\title{
The societal impact of the emerging quantum technologies: a renewed urgency to make quantum theory understandable
}

\author{
Pieter E. Vermaas ${ }^{1}$
}

Published online: 24 July 2017

(c) Springer Science+Business Media B.V. 2017

\begin{abstract}
This paper introduces the special issue The societal impact of the emerging quantum technologies as a contribution to a more inclusive societal debate on quantum technologies. It brings together five contributions. Three are authored by quantum technology researchers who give explorations of the possible impacts of quantum technologies on science, industry and society. The fourth contribution discusses within the framework of responsible research and innovation, the ways in which quantum technologies and the societal debate about them are presented in European policy documents. The final contribution analyses how the popularisation of quantum theory for wider audiences has evolved, and can be improved, with the emergence of quantum technologies. This introduction is also a call for a renewed effort to make quantum theory understandable. A preamble to a societal debate about quantum technologies is that all stakeholders understand these technologies to a reasonable degree, and the current framing of quantum theory as enigmatic in not helpful to meeting this. It is argued that philosophers of physics can help overcome this framing by explaining how quantum theory and quantum technologies are similar to every-day descriptions and technologies.
\end{abstract}

Keywords Societal debate on quantum technologies · Responsible research and innovation · Philosophy of physics · Popularisation of quantum theory $\cdot$ Impact of quantum computing - Impact of quantum cryptography · Quantum Manifesto

Pieter E. Vermaas

p.e.vermaas@tudelft.nl

1 Philosophy Department, Delft University of Technology, Jaffalaan 5, 2628BX Delft, The Netherlands

\section{Introduction}

Quantum theory is rapidly transforming from a specialist theory in physics to a challenging source of new technologies for science, industry and government. Research on quantum technologies such as quantum computing, quantum communication, quantum sensing and quantum cryptography is speeding up, and has already created first operational and commercially available applications. Governmental and corporate efforts to further scale up this research and development are in place or on their way. This growth of research on quantum technologies calls for a societal debate to explore and assess the impacts that quantum technologies will have on science, industry, people and society. The development of quantum technologies propels but is apart from the first applications still in a preliminary phase in which research groups compete on first discoveries, patent positions and academic honour. Governmental actors have joined by their strategic visions and funding schemes (e.g., the Quantum Technologies Flagship initiative by the European Commission 2016). And industry is defining and developing their positions and charting the economic prospects (as in, e.g., Economist 2017). It is now time that other stakeholders join too, for becoming part of discussions on quantum technologies and of the decisions about what we want with these technologies. This special issue of Ethics and Information Technologies on the societal impact of the emerging quantum technologies is meant as a first contribution to this more inclusive societal debate. A debate on quantum technologies between researchers, governments, industry, ethicists, social scientists and stakeholders in society is currently not taking place, and with its contributions this special issue aims to help giving it a basis. It offers a first exploration of the possible impacts of quantum technologies and it gives first analyses of how 
quantum theory and quantum technologies are presented by researchers and funding agencies. It is also a call to philosophers of physics for a renewed effort to make quantum theory understandable. A recurrent observation in the contributions to this special issue is that quantum technology is hard to understand by stakeholders due to the counterintuitive nature of how quantum theory describes elementary particles and light. This observation may imply that stakeholders cannot yet fully participate in a societal debate on quantum technologies, since a preamble to this debate is that all participants can understand quantum technologies to a reasonable degree. I believe philosophers can help with providing this understanding. In philosophy of physics quantum theory has been extensively analysed for the way in which it describes reality or represents quantum systems. By these analyses philosophers of physics can now enable stakeholders to join the societal debate on quantum technologies.

After expanding on this call to philosophers of physics in "Quantum theory as the enigmatic source of quantum technologies" I discuss in "The contributions and the issues" the contributions to this special issue and the issues they identify for a societal debate on quantum technologies. Three of the five contributions are authored by researchers who are developing quantum technologies, one originates for the field of technology assessment, and one from philosophy of physics. I end with an outlook.

\section{Quantum theory as the enigmatic source of quantum technologies}

The potential impacts of quantum technologies warrant already paying good attention to these technologies. Prospected applications such as quantum computing, quantum communication and quantum cryptography are assumed to have major effects on the scientific and engineering problems we can take up, and on the security of governmental and commercial data and communication. As such the developments that quantum technologies may bring are as innovative and disruptive as other grand technologies such as nanotechnology and artificial intelligence. And given that these later two programmes are topic of societal debates, we better also discuss quantum technologies. Present-day thinking about technology development moreover calls for more pro-active engagements of stakeholders. The times are past of giving societal or ethical evaluations of new technologies when researchers, engineers and industry have made them ready to market. Today society and its funding agencies adopt responsible research and innovation approaches (e.g., Von Schomberg 2011; Owen et al. 2012; Van den Hoven et al. 2014; Koops et al. 2015, see also the special issue contribution by Coenen and Grunwald), in which all stakeholders already in the earlier phases of research participate in discussions of how developments should be guided towards the technologies we want. And in the related design for values approaches (e.g., Friedman et al. 2006; Van den Hoven et al. 2015) we immediately include our moral and societal values in the development of these new technologies. These present-day approaches presuppose that stakeholders understand quantum technologies, that we can explore what quantum technologies may bring, and that we can decide about what we judge to be responsible and in line with our values. With that knowledge and with those decisions we can then shape the governmental funding schemes we put in place for developing the quantum technologies we want and respond to or intervene if corporate efforts are heading in opposite directions.

Starting a societal debate on quantum technologies brings the question of whether it will bring novelties as compared to earlier debates on grand technologies. It is my contention that it will. Apart from the novelties that specific quantum technologies may bring-think of quantum cryptography and its impact on data security-the debate will bring the challenge to stakeholders to understand quantum technologies. A societal debate on quantum technologies and the approach of responsible research and innovation have, as said, as a preamble that all stakeholders have this understanding. Quantum technologies are however technologies that make quantum theory technologically applicable, and quantum theory is up to this day framed as an enigmatic theory whose counterintuitive descriptions of the physical realm are difficult to master. Physicists and quantum technology researchers may already have a hard time understanding these descriptions, sharing this understanding with other stakeholders may prove to be even more difficult. Hence, the knowledge gap between research and society may not be so easily overcome in the case of quantum technologies, hampering an open debate. It is also my contention that this gap can be bridged with the help of philosophers of physics, who have extensive experience with making quantum theory understandable (see also the special issue contribution by Grinbaum). Hence, philosophy should not only be involved in a social debate on quantum technologies through its branches of ethics and philosophy of technology; a novelty of this debate is that also philosophers of physics should contribute by realising its preamble of making these technologies intelligible to all stakeholders.

Quantum theory is the theory that describes the physics of elementary particles and light. It is also a theory that since its inception in the early twentieth century has acquired the status of an enigmatic theory. As the theory of relativity, quantum theory was presented as giving descriptions of reality that are fundamentally different to 
the descriptions that the physical theories of the previous nineteenth century provide. And by presenting those nineteenth century physical theories as classical theories that are intuitively clear, quantum theory and its descriptions of reality were framed as counterintuitive and unintelligible. This framing persists till this day and the list of counterintuitive and enigmatic features of quantum theory is impressive. Whereas by classical physics physical systems are typically either particles or waves, quantum theory allows that the behaviour of physical systems alternates between that of particles and that of waves. And where by classical physics particles have typically well-defined positions and velocities, quantum theory introduces uncertainty relations for the positions and velocities (momenta, to be precise) of systems. The wave behaviour of systems in quantum theory moreover allows that the physical states of systems can be combinations (superpositions) of states that are classically quite separate (as Schrödinger's cat that is in a state that combines the states of being alive and being dead), that a single particle can simultaneously follow different trajectories and then as waves interfere with itself (as in the double-slit experiment), and that the states of far-away systems can be connected (entangled) in a way that allows that a change of the state in one system leads to instantaneous changes in the states of the other systems. Finally quantum mechanics replaces the deterministic worldview of classical physics in which systems the behaviour of systems is with certainty fixed through time, with a worldview by which systems behave in a fundamentally stochastic way.

Understanding quantum theory by analysing how its descriptions of elementary particles and light deviate from descriptions as given by the earlier classical physics makes academic sense, of course. It has, moreover, led to a rich and fundamental research tradition in physics and philosophy, that started with early discussions by the founding fathers of quantum theory (see, for instance, Bacciagaluppi and Valentini 2009), to proofs of how quantum theory violates classical and relativistic intuitions (e.g., no local hidden-variable theories: Einstein et al. 1935; Von Neumann 1955; Bell 1987; Redhead 1987) and to elaborate attempts to capture how quantum theory nevertheless describes physical reality (e.g., interpretations of quantum theory as, for instance, Bohm 1952; Everett 1957). Yet, focussing on the counterintuitive aspects makes less sense when quantum theory and its application in quantum technology have to be made intelligible beyond the communities of physicists and philosophers. Taking quantum theory and quantum technology as enigmatic introduces then a barrier for stakeholders in science, industry and society to discuss quantum technologies. For these stakeholders it would instead be more useful to present quantum theory and its applications as novel though understandable. And when quantum technologies become operational and are requiring larger numbers of quantum engineers, it again would be more useful to present quantum theory as a normal theory that can be mastered and applied just as any other scientific theory. That such a change in framing is possible is illustrated in the contribution by Grinbaum when he argues that quantum theory is increasingly introduced to a wider audience not by enigmatic metaphors such as Schrödinger's cat, but by the technological effects that can be realised by quantum theory. It can also be observed by a shift in philosophy of physics to see quantum theory less as a counterintuitive theory about reality and more as a novel theory about information (e.g., Fuchs 2010). And one can imagine that when quantum theory by its technological applications becomes a regular ingredient in the curricula of secondary schools and academic programmes, this theory will eventually be presented as a regular physical theory with a straightforward meaning and interpretation (Vermaas 2005). Philosophers of physics can help in speeding up this change in framing quantum theory and thus in enabling all stakeholders to join the social debate on quantum technologies. Philosophers of physics have analysed in detail how quantum theory describes elementary particles and light, and can tell not only at what points these descriptions are less intelligible, but also to what extent quantum theory and quantum technologies are similar to every-day descriptions and technologies.

\section{The contributions and the issues}

The five contributions to this special issue on the societal impact of quantum technologies approach the topic in different ways. Three contributions are by researchers in quantum technologies and these are focussed on defining and exploring the impact of quantum technologies on science, industry and society. As many of these technologies are far from being realised, the authors of these three contributions proceed cautiously, avoid speculation, and warn for too high expectations. In the first contribution David P. DiVincenzo chooses solid ground for his exploration by placing the development of quantum technologies in a larger scientific development, and by exploring what these technologies can deliver through critically analyzing the promises presented in the European Quantum Manifesto (De Touzalin et al. 2016). In the second contribution Matthias Möller and Cornelis Vuik approach quantum computing within the context of scientific computation, and argue that quantum computing is a next development that, compared to computing with existing classical computers, will widen the field's envelop: quantum computers will allow computational scientists to pick up some new problems and address more efficiently a number of existing problems. Ronald de Wolf, in the third contribution, takes a more 
direct approach by describing immediately the potential societal impacts of quantum technologies in the domains of cryptography, search algorithms and the simulation of the behaviour of molecules and materials, and by exploring the ethical issues these impacts may bring.

The two further contributions are not focussed on the possible impacts of quantum technologies but on how stakeholders other than quantum researchers and policy makers can be drawn in a societal debate on these technologies and their impacts. The fourth paper by Christopher Coenen and Armin Grunwald discusses the ways quantum technologies and the societal debate about them, are presented in policy documents from Germany and the United Kingdom, and in the already mentioned European quantum manifesto. This presentation is compared to the way in which nanotechnologies were initially announced and debated. Coenen and Grunwald observe similarities between the two cases, such as the guiding role policymakers play in propelling the development of both types of technologies, and the grand claims about the positive developments these technologies are supposed to bring to science, industry and society. A difference that Coenen and Grunwald identify is that quantum technologies are not or less associated with more utopian visions about the world or about humanity, as nanotechnologies were initially associated with transhumanism. Another difference, relevant to this special issue, is that the emerging societal debate on quantum technologies is in the policy documents not always described as an open twoway exchange. Whereas current responsible research and innovation approaches aim at including stakeholders in discussions about new technologies in the early phases of the development of these technologies, the "exchange" that the policy documents on quantum technologies propose may still be a mere dissemination to lay persons of what quantum technologies will be. Coenen and Grunwald argue for a full-blown responsible research and innovation debate on quantum technologies in which all stakeholders participate, and give recommendations about how to arrive at such a debate.

The fifth and final contribution by Alexei Grinbaum from the perspective of philosophy of physics brings us back to the discussed preamble to drawing in all stakeholders in a full-blown societal debate on quantum technologies. Grinbaum describes developments in the ways in which understanding of quantum theory is relayed to broader audiences through popularisation. Where in the earlier days of quantum theory physicists and philosophers introduced this theory by either experimental facts that cannot be explained by classical theories or by novel theoretical assumptions such as superposition or entanglement of physical states, a current approach is presenting quantum theory through the tasks that can be taken up with this theory, as say in cryptography. One could conclude that this latter approach may enable stakeholders to understand quantum technologies without getting caught in the enigmas of quantum theory, thus realising the preamble to a societal debate. Yet Grinbaum sees also limitations to this approach since it does not inform stakeholders in what sense quantum theory and quantum technologies are novel, and since it does not explain why quantum technologies can do the tasks for which they are used. Stakeholders can therefore not share the understanding that quantum researchers have, which may hamper public trust in these researchers. Grinbaum then develops means for arriving at this shared understanding and trust.

The quantum researchers who contributed to this special issue mention the counter-intuitiveness of quantum theory. The contribution by Möller and Vuik contains more detailed explanation of quantum theory, yet by introducing quantum technologies mainly through the tasks they can carry out the three first contributions fit within Grinbaum's analysis. Taking this as just another illustration of the one-way dissemination that Coenen and Grunwald observe would however be unfair, since this special issue is about exploring the impacts of quantum technologies, and in the three first contributions the authors do critically reflect on how research on these technologies advances and is presented. And by their reflections they seem to warn us for a looming quantum divide between those who develop quantum technologies and those who are not, calling for the two-way exchange that Coenen and Grunwald propose. DiVincenzo, for instance, is critical about the technological promises that are made to society in the European Quantum Manifesto. He analyses that this research will remain in the coming years primarily an enterprise within quantum science, and may eventually lead to quite different technologies, calling for a more active monitoring role for society that cuts through the knowledge divide between quantum scientists and society. De Wolf, in turn, warns that by the huge current investments of mainly US companies and governmental agencies quantum computing may easily become monopolised by a few actors at the expense of others. He pleads for creating also publically available quantum computing facilities, which cut through the divide between the haves and have-nots. Finally, Möller and Vuik note that for fully exploiting the new possibilities that quantum computing can bring, large groups of students have to be educated in programming these computers, meaning that understanding of quantum technologies should be made available beyond the community of quantum technology researchers.

The impact of quantum technologies that are in more detail described in the three contributions by quantum researchers concern those of quantum computing and of quantum communication and cryptography. The impact of quantum computing on society is sketched as emerging indirectly through the advancements it can bring to 
scientific research. Quantum computers will not radically change science or fully replace research done with classical digital computers. Rather quantum computing can outperform classical digital computing in addressing specific computational tasks, as described in more detail by Möller and Vuik, and gives therefore scientists tools for taking up a number of new scientific challenges and addressing existing ones more efficiently. Challenges that are identified include faster search and optimisation algorithms and the simulation of the behaviour of molecules and of other quantum systems. And when quantum computing can tackle these challenges, the impacts will become available to society. The promises of quantum algorithms lie in finding optimal solutions to complex modelling and design problems, leading to for instance better predictions of the behaviour of fluids and gasses (e.g., of floods) and to more efficient products (e.g., aircraft that consume less energy). Mentioned promises of simulation are the search for and design of new materials for medicine (drugs), engineering (e.g., construction materials) and agriculture (e.g., fertilisers).

The societal impact of quantum communication and cryptography is sketched as being more direct and also as more problematic. Shor's (1997) algorithm for finding the prime numbers that factor large integers was an early result that demonstrated that quantum computing could perform tasks faster than classical computers can do. This result also showed that quantum computing, when made available, would compromise current encryption techniques that nowadays secure communication between for instance financial institutions and governmental organisations. And although quantum computing may still not yet be advanced enough to actually decrypt current communication, the mere fact that it could does have already its effects nowadays. As argued by De Wolf this promise makes it meaningful to intercept and store current encrypted communication and data bases, and decrypt them at the time quantum computing does exist. This would make that state and company secrets that are nowadays sufficiently protected become eventually available to those who collected the data. De Wolf shows that quantum technology also gives a response to this potential breach in the form of quantum encryption techniques, called post-quantum cryptography. Still, this need to rethink and adjust current encryption techniques demonstrates that quantum technologies have already nowadays an impact on society. This impact may even become larger by other quantum technologies, as the above mentioned search algorithms that enable more intrusive analyses of the communication and data of individuals and other actors, and the possibility of creating with quantum technologies a communication network that may be fully secure to classical and quantum decryption. The European Quantum Manifesto (De Touzalin et al. 2016) takes such a secure network as one of the results quantum technologies will deliver, and calls it a quantum internet, signalling that it may be available to all. Yet, one can envisage also such networks for only governmental agencies, international companies or even organised crime, enabling another quantum divide between actors who can communicate securely and others whose communication is made transparent by quantum search algorithms. When focussing on quantum communication and cryptography, society has indeed reason, as noted by DiVincenzo, to consider the impact of quantum technologies as potentially negative.

\section{Outlook}

This special issue on The societal impact of the emerging quantum technologies is meant as a contribution to a more inclusive societal debate on quantum technologies. It offers a first exploration of the possible impacts of quantum technologies and it gives first analyses of how quantum theory and quantum technologies are presented by researchers and funding agencies. It is also a call to philosophers of physics for a renewed effort to make quantum theory understandable. A preamble to a societal debate is that all participants understand quantum technologies to a reasonable degree, and the current framing of quantum theory as enigmatic in not helpful for arrive at this understanding. I argued that philosophers of physics can help overcome this framing by explaining how quantum theory and quantum technologies are similar to every-day descriptions and technologies.

In this special issue Coenen and Grunwald argue for organising the societal debate as an open dialogue between stakeholders on individual quantum technologies instead of as one grand debate on quantum technology in general. The debate, or better said, the debates can then be focussed on particular technologies, for instance, on quantum communication and cryptography, and their impacts on society. A further split can be made between issues that are raised by the introduction of quantum technologies and issues that are raised by an ongoing use of these technologies. Some of the issues I discussed under the label of quantum divide may surface in the transitional period in which first quantum technologies are introduced, and disappear when they become generally available and mainstream. The debate on such issues can focus on the transitional period, say when the first quantum computers become operational, and define responses to consequence we want to avoid, say by making quantum computing quickly publically available. Other issues may however have a more permanent impact on society, as for instance the divide that can emerge when secure quantum communication is made available to only a few stakeholders. Such permanent issues may even be more relevant to discuss and address now given that the 
development of quantum technologies is still in an early phase and can be guided in the direction we want.

I end with two final observations. The first is that the exploration of the potential impacts of quantum technologies is itself in an early phase. The quantum technologies to which attention is given in this special issue are the ones that are already defined to some degree. Yet new quantum technologies may emerge and others are in the making, as say the technologies related to graphene or to quantum teleportation. Hence, a societal debate indeed should be an open one, allowing for new quantum technologies to be included and new stakeholders to join. Finally, it is sometimes remarked, also in this special issue, that the impacts that quantum technologies may bring could equally have been caused by other non-quantum technologies. For instance, it can be noted that Shor's algorithm for finding prime numbers of integers corrupts existing encryption techniques, but that other algorithms not relying on quantum computing could have done so as well. This remark may be true, yet does not take away the urgency to discuss these impacts in a societal debate on quantum technologies. That debate should still be about whether or not we want these impacts.

Acknowledgements I want to thank Jeroen van den Hoven, Editorin-Chief of Ethics and Information Technologies, for inviting me to compose this special issue, and to praise the authors of its contributions for taking up the challenge to reflect in this early phase on the societal impact of the emerging quantum technologies.

\section{References}

Bacciagaluppi, G., \& Valentini, A. (2009). Quantum theory at the crossroads: Reconsidering the 1927 Solvay conference. Cambridge: Cambridge University Press.

Bell, J. S. (1987). Speakable and unspeakable in quantum mechanics. Cambridge: Cambridge University Press.

Bohm, D. (1952). A suggested interpretation of the quantum theory in terms of 'hidden' variables, I and II. Physical Review, 85, 166-193.

De Touzalin, A., Marcus, C., Heijman, F., Cirac, I., Murray, R., \& Calarco, T. (2016). Quantum manifesto: A new era of technology.
QUTE-EUROPE. http://qurope.eu/manifesto. Accessed 12 May 2017.

Economist (2017). Technological quarterly: Quantum devices. Accessed 11 March 2017.

Einstein, A., Podolsky, B., \& Rosen, N. (1935). Can quantummechanical description of physical reality be considered complete? Physical Review, 47, 777-780.

European Committee (2016). European Commission will launch $€ 1$ billion quantum technologies flagship. https://ec.europa. eu/digital-single-market/en/news/european-commission-willlaunch-eu1-billion-quantum-technologies-flagship. Accessed 12 May 2017.

Everett, H. (1957). 'Relative state' formulation of quantum mechanics. Reviews of Modern Physics, 29, 454-462.

Friedman, B., Kahn, P. H. Jr., \& Borning, A. (2006). Value sensitive design and information systems. In P. Zhang \& D. Galletta (Eds.), Human-computer interaction in management information systems: Foundations (pp. 348-372). Armonk, NY: M.E. Sharpe.

Fuchs, C. A. (2010). Coming of age with quantum information: Notes on a Paulian idea. Cambridge: Cambridge University Press.

Hoven, J. Van den, Doorn, N., Swierstra, T., Koops, B.-J., \& Romijn, H. (Eds.). (2014). Responsible innovation 1: Innovative solutions for global issues. Dordrecht: Springer.

Hoven, J. Van den, Vermaas, P. E., \& Poel, I. Van de (Eds.). (2015). Handbook of ethics, values and technological design. Dordrecht: Springer.

Koops, B.-J., Oosterlaken, I., Romijn, H., Swierstra, T., \& Hoven, J. Van den (Eds.). (2015). Responsible innovation 2: Concepts, approaches, and applications. Dordrecht: Springer.

Owen, R., Macnaghten, P., \& Stilgoe, J. (2012). Responsible research and innovation: From science in society to science for society, with society. Science and Public Policy, 39, 751-760.

Redhead, M. (1987). Incompleteness, nonlocality, and realism. Oxford: Oxford University Press.

Shor, P. W. (1997). Polynomial-time algorithms for prime factorization and discrete logarithms on a quantum computer. SIAM Journal on Computing, 26, 1484-1509.

Vermaas, P. E. (2005). Technology and the conditions on interpretations of quantum mechanics. British Journal for the Philosophy of Science, 56, 635-661.

Von Neumann, J. (1955). Mathematical foundations of quantum mechanics. Princeton: Princeton University Press.

Von Schomberg, R. (2011). Towards responsible research and innovation in the information and communication technologies and security technologies fields. European Commission. https://philpapers.org/archive/VONTRR.pdf. Accessed 24 May 2017. 